\title{
L'homologation progressive : un débat ouvert progressif s'impose
}

$\mathrm{L}$ e retrait mondial du rofécoxib (Vioxx) a marqué la fin d'une époque d'innocence relative chez les consommateurs. Il a démontré sans équivoque que, contrairement à d'autres «biens" de consommation, les produits pharmaceutiques ne peuvent être régis par le profit. L'élément moteur doit plutôt être un système de réglementation transparent et axé sur les patients qui garantisse l'innocuité et l'efficacité des médicaments avant et après leur mise en marché.

Dans le sillage du fiasco du rofécoxib, Santé Canada et les organismes de réglementation du monde entier révisent leur façon d'approuver et de surveiller les médicaments en appliquant «l'approbation progressive» ${ }^{1}$. Poussé par une étude marquante de l'innocuité des médicaments réalisée par l'Institute of Medicine, le Sénat américain a adopté récemment un projet de loi pour augmenter considérablement le pouvoir d'évaluation des médicaments de la Food and Drug Administration (FDA) des États-Unis et a notamment accru considérablement ses pouvoirs de surveillance postcommercialisation.

Santé Canada affirme que sa propre version de l'approbation progressive comprend un virage patients, la collecte et le résumé de données probantes sur les médicaments dès leur mise au point et l'amélioration de la surveillance postcommercialisation ${ }^{2}$. Santé Canada s'emploie actuellement à recueillir de l'information et à rédiger des énoncés de position pour consultation.

En ce moment la mise en place de l'approbation progressive exigera une importante refonte de la législation et des règlements afin d'améliorer l'approbation et la surveillance postcommercialisation. Il se peut aussi que le seuil d'approbation de certains nouveaux médicaments soit plus bas, mais qu'en revanche les obligations d'évaluer continuellement les médicaments après leur commercialisation soient plus rigoureuses. Du côté positif, ce changement pourrait accélérer l'accès à de nouveaux traitements, mais du côté négatif, des médicaments dangereux risquent peutêtre de franchir le filet de la réglementation. Le compromis est assez évident, mais est-il nécessaire? Et, plus important encore, à qui incomberont les décisions?

Deux voix dominent actuellement les discussions sur le processus de changement : celle de l'industrie pharmaceutique et celle de Santé Canada. Or, ces intervenants sont certes importants, mais il ne sont pas les seuls; la convergence de leurs efforts est beaucoup trop étroite et, peut-être, intéressée. Les professionnels de la santé, les experts et la population du Canada ne sont nulle part représentés.

Nous sommes d'avis que la mise en place d'une véritable réforme exigera de Santé Canada et du gouvernement qu'ils soient blindés contre l'influence omniprésente des lobbyistes, ce qui pourrait se faire en invitant les Académies canadiennes des sciences (notre version des National Academies of Science des États-Unis qui supervisent l'Institute of Medicine) ou l'Académie canadienne des sciences de la santé (notre version de l'Institute of Medicine des États-Unis) à + créer un groupe d'experts chargé de conseiller le Parlement. Dans le contexte d'un processus ouvert et transparent, il faudrait désigner des représentants des principaux groupes intéressés du Canada, y compris de la population, ainsi que d'organismes de réglementation de l'étranger. Ces intervenants ont beaucoup à ajouter à la symphonie à deux de l'industrie et de Santé Canada.

Le groupe d'experts pourrait examiner d'abord le système actuel pour voir si, par exemple, une séparation plus marquée entre les fonctions d'approbation des médicaments et de surveillance postcommercialisation, ainsi des pouvoirs accrus de surveillance des médicaments, nous serviraient mieux. L'autorisation précommercialisation n'exige actuellement que deux études de phase III réalisés sur des patients très sélectionnés. Ces études produisent trop peu de données chez trop peu de patients. Dans le contexte du processus d'approbation, le groupe d'experts pourrait insister pour que les médicaments fassent l'objet d'études pratiques d'envergure. Le Canada pourrait aussi utiliser sa force unique dans le domaine des examens systématiques avec le collectif Cochrane et des centres de pratique factuelle. De telles études peuvent produire des résultats significatifs, comme l'a démontré récemment l'avertissement de risque cardiaque joint à la rosiglitazone (Avandia) par la FDA des États-Unis ${ }^{3}$. Le groupe d'experts pourrait décider de la meilleure façon d'intégrer cette expertise à un système de préalerte.

Dans l'intérêt de l'efficience, le groupe d'experts pourrait aussi évaluer les avantages de l'intégration des organismes de réglementation au Canada, aux États-Unis et en Europe. Une telle harmonisation pourrait produire des normes rigoureuses uniformes et généralisées, éviter le double emploi, accroître la confiance et peutêtre, éventuellement, déboucher sur des domaines d'expertise synergiques en contenu clinique ou en méthodologies de recherche.

Dans l'ensemble, le cas du rofécoxib et, plus récemment, du tégasérod (Zelnorm $)^{4}$ démontrent que les processus décisionnels doivent être plus ouverts et transparents. Les sociétés pharmaceutiques doivent donner accès en temps opportun et de façon utilisable à tous les renseignements dont elles disposent sur l'innocuité des médicaments, peut-être en affichant les principaux résultats dans des registres publics sur les études. Les divers paliers de gouvernement doivent collaborer davantage afin que les chercheurs et les intéressés puissent avoir accès à tous les renseignements nécessaires. Un futur groupe d'expert devra tenir compte de beaucoup de facteurs.

Nous croyons que l'approbation progressive de Santé Canada, sans ouverture progressive du débat, a peu de chance d'offrir une avenue de progrès véritable. La conception du cadre de réglementation des médicaments est trop importante pour qu'on s'en remette aux fonctionnaires et à l'industrie seulement. Il faut la leur enlever et la soumettre à une discussion franche et ouverte des besoins de la société.

Paul C. Hébert, Rédacteur en chef, JAMC

Avec l'équipe de rédaction de l'éditorial (Matthew Stanbrook, Barbara Sibbald, Ken Flegel, Noni MacDonald et Amir Attaran)

Intérêts concurrents : Noni MacDonald est membre de l'Académie canadienne des sciences de la santé. Aucuns déclarés par les autres membres de l'équipe de rédaction.

\section{RÉFÉRENCES}

I. Kondro W. Health Canada proposes new regulatory regime for drugs. JAMC 2007;176:I26I-2.

2. Yeates N, Lee DK, Maher M. Health Canada's progressive licensing framework. JAMC 2007; I76:1845-7.

3. Nissen SE, Wolski K. Effect of rosiglitazone on the risk of myocardial infarction and death from cardiovascular causes. N Engl J Med. Publication électronique le 2I mai 2007 avant la version imprimée. Disponible à : http://content.nejm.org/cgi /content/full/NEJMoa07276I (consulté le 22 mai 2007).

4. Wright JM. Progressive drug licensing: An opportunity to achieve transparency and accountability? JAMC 2007;176:1848-9. 